\title{
Generalized Common Fixed Point Theorem for Four Self Mappings in b- Metric Space
}

\author{
Akash singhal ${ }^{1 *}$, Rajesh Kumar Sharma, Anil Agrawal \\ ${ }^{1}$ Department of Mathematics, Institute of Technology \& Management, ITM Campus, Gwalior, India \\ ${ }^{1}$ Research Scholar MGCGV Chitrakoot Satna, India \\ ${ }^{2}$ Department of Mathematics, Govt. Holkar Science College, Indore, India \\ ${ }^{3}$ Faculty of Science \& Environment MGCGV, Chitrakoot, Satna, India
}

Available online at: www.isroset.org

Accepted 27/Jun/2018, Online 30/Aug/2018

\begin{abstract}
In this paper we have tried to prove a common fixed point theorem of four self-mappings satisfying a generalized contractive condition in b-metric space. Our result extend several comparable pre-existing results in literature. We have also given an example that shows the applicability and validity of our result.
\end{abstract}

Keywords- Fixed point; Common Fixed point; Cauchy Sequence; compatible mapping; b- Metric space

2000 classification: $47 \mathrm{H} 10,54 \mathrm{H} 25$

\section{INTRODUCTION AND PRELIMINARIES}

The idea of b-metric space was introduced by Bourbaki [6] and Bakhtin[3]in 1989. Czerwik[8] give an axiom which was weaker than triangle inequality and formally defined a b-metric space with a view of generalization the Banach contraction mapping theorem. Since then several research paper have dealt with fixed point theory for single and multi-valued mapping in b- metric spaces .Pacurar [12] extended the fixed point theorem in b-metric space. Many authors in their work have used the bmetric spaces in which b- metric function is continuous, but the techniques used here can be employed in the setup of discontinuous -b metric spaces.in this paper we focused on Ćirić and Hardy-Rogers type contractions and present a common fixed point in b-metric spaces.

Our result extended and improve the result of J.R. Roshan, N.Shobkolaei, S.Sedghi and M. Abbas [10] and several comparable result that exist in literature, Following definitions and result will be needed in the sequel.

Definition 1.1: Let $X$ be a non-empty set and $s \geq 1$ be a given real no., function $d: X x X \rightarrow R^{+}$is a b-metric iff for each $x, y, z \in X$ if following conditions are hold ;

(1) $d(x, y)=0$ iff $x=y$

(2) $d(x, y)=d(y, x)$

(3) $\mathrm{d}(\mathrm{x}, \mathrm{z}) \leq \mathrm{s}[\mathrm{d}(\mathrm{x}, \mathrm{y})+\mathrm{d}(\mathrm{y}, \mathrm{z})]$

A pair $(X, d)$ is called a $b$-metric space.

Definition 1.2: Let $(X, d)$ be a b-metric space then a sequence $\left\{x_{n}\right\}$ in $X$ is called

(i) Convergent if and only if there exist $x \in X$ such that $d\left(x_{n}, x\right) \rightarrow 0$ as $n \rightarrow \infty$.

(ii) Cauchy if and only if $d\left(x_{n}, x_{m}\right) \rightarrow 0$ as $m, n \rightarrow \infty$.

(iii) The b-metric space $(\mathrm{X}, \mathrm{d})$ is complete if every Cauchy sequence is convergent.

Definition 1.3: $(X, d)$ be a b-metric space. A pair $\{f, g\}$ is said to be compatible if and only if $\quad \lim n \rightarrow \infty d$ $\left(f g x_{n}, g f x_{n}\right)=0$ whenever $\left\{x_{n}\right)$ is a sequence in $X$ such that $\lim n \rightarrow \infty f x_{n}=\lim n \rightarrow \infty \mathrm{gx}_{n}=t$ for some $t \in X$

Lemma 1.4: Let $(X, d)$ be a b-metric space with $k \geq 1$, suppose that $\left\{x_{n}\right\}$ and $\left\{y_{n}\right\}$ are $b$-convergent such that to $x$ and $y$ respectively .then we have 
$\frac{1}{k^{2}} d(x, y) \leq \lim n \rightarrow \infty \inf d\left(x_{n}, y_{n}\right) \leq \lim n \rightarrow \infty \sup d\left(x_{n}, y_{n}\right) \leq k^{2} d(x, y)$

In Particular, if $\mathrm{x}=\mathrm{y}$, then we have $\lim \mathrm{n} \rightarrow \infty \mathrm{d}\left(\mathrm{x}_{\mathrm{n}}, \mathrm{y}_{\mathrm{n}}\right)=0$ moreover for each $\mathrm{z} \in \mathrm{X}$ we have

$\frac{1}{k} d(x, z) \leq \lim n \rightarrow \infty \inf d\left(x_{n}, z\right) \leq \lim n \rightarrow \infty \sup d\left(x_{n}, z\right) \leq k d(x, z)$

Lemma 1.5: Let $(X, d)$ be a b-metric space. If there exist two sequence $\left\{x_{n}\right\}$ and $\left\{y_{n}\right\}$ such that $\lim n \rightarrow \infty d\left(x_{n}, y_{n}\right)=0$.

Wherever $\left\{x_{n}\right\}$ is a sequence in $X$ such that $\lim n \rightarrow \infty x_{n}=t$ for some $t \in X$ then $\lim n \rightarrow \infty y_{n}=t$.

\section{MAIN RESULT}

Theorem (2.1) : suppose that F,G,S and $T$ are self-mapping on a complete b-metric space $(X, d)$ such that $F(X) \subseteq T(X), G(X)$ $\subseteq \mathrm{S}(\mathrm{X})$, if

$(2.1) d(F x, G y) \leq \frac{a_{1}}{k^{4}}\left[a_{2} \max \left\{d(S x, T y), d(F x, S x), d(G y, T y), \frac{1}{2}(d(S x, G y)+d(F x, T y))\right\}+\left(1-a_{2}\right)\left\{\max \left(d^{2}(S x, T y)\right.\right.\right.$,

$\left.\left.\left.d(F x, S x) d(G y, T y), d(S x, G y) \cdot d(F x, T y), \frac{d(F x, S x) d(F x, S y)}{2}, \frac{d(S y, G y) d(S x, G y)}{2}\right)\right\}^{1 / 2}\right]$.

Where $\mathrm{a}_{1} \in(0,1)$ and $0 \leq \mathrm{a}_{2} \leq 1$

Holds for each $\mathrm{x}, \mathrm{y} \in \mathrm{X}$ with $0<\mathrm{a}_{1}<1$ then $\mathrm{F}, \mathrm{G}, \mathrm{S}$ and $\mathrm{T}$ have a unique common fixed point in $\mathrm{X}$ provided that $\mathrm{S}$ and $\mathrm{T}$ are continuous and pairs $(\mathrm{F}, \mathrm{S})$ and $(\mathrm{G}, \mathrm{T})$ are compatible.

Proof: Let $x_{0} \in X$ as $F(X) \subseteq T(X)$ \& $x_{1} \in X$ s.t $F\left(x_{0}\right)=T x_{1}$. since $G x_{1} \in S(X)$, we can chose $x_{2} \in X$ such that $G_{1}=S x_{2}$ in general $\mathrm{x}_{2 \mathrm{n}+1}$ and $\mathrm{x}_{2 \mathrm{n}+2}$ are chosen in $\mathrm{X}$ such that $\mathrm{Fx}_{2 \mathrm{n}}=\mathrm{Tx}_{2 \mathrm{n}+1}$ and $\mathrm{Gx}_{2 \mathrm{n}+1}=\mathrm{Sx}_{2 \mathrm{n}+2}$ define a sequence $\left\{\mathrm{y}_{\mathrm{n}}\right\}$ in $X$ such that $\mathrm{y}_{2 \mathrm{n}}=\mathrm{Fx}_{2 \mathrm{n}}=$ $\mathrm{Tx}_{2 \mathrm{n}+1}$ and $\mathrm{y}_{2 \mathrm{n}+1}=\mathrm{Gx}_{2 \mathrm{n}+1}=\mathrm{Sx}_{2 \mathrm{n}+2}$ for all $\mathrm{n} \geq 0$ Now we show that $\left\{\mathrm{y}_{\mathrm{n}}\right\}$ is a Cauchy sequence, consider

$$
\begin{aligned}
& d\left(y_{2 n}, y_{2 n+1}\right)=\left(F x_{2 n}, G x_{2 n+1}\right) \leq \frac{a_{1}}{k^{4}}\left[a _ { 2 } \operatorname { m a x } \left\{d\left(S x_{2 n}, T x_{2 n+1}\right), d\left(F x_{2 n}, S x_{2 n}\right), d\left(G x_{2 n+1}, T x_{2 n+1}\right),\right.\right. \\
& \left.\frac{1}{2}\left(d\left(S x_{2 n}, G x_{2 n+1}\right)+d\left(F x_{2 n}, T x_{2 n+1}\right)\right)\right\}+\left(1-a_{2}\right)\left\{\operatorname { m a x } \left(d^{2}\left(S x_{2 n}, T x_{2 n+1}\right), d\left(F x_{2 n} S x_{2 n}\right) d\left(G x_{2 n+1}, T x_{2 n+1}\right),\right.\right. \\
& \left.\left.\left.d\left(S x_{2 n}, G x_{2 n+1}\right) \cdot d\left(F x_{2 n}, T x_{2 n+1}\right), \frac{d\left(F x_{2 n}, S x_{2 n}\right) d\left(F x_{2 n}, S x_{2 n+1}\right)}{2}, \frac{d\left(S x_{2 n+1}, G x_{2 n+1}\right) d\left(S x_{2 n}, G x_{2 n+1}\right)}{2}\right)\right\}^{1 / 2}\right] \\
& =\frac{a_{1}}{k^{4}}\left[a_{2} \max \left\{d\left(y_{2 n-1}, y_{2 n}\right), d\left(y_{2 n}, y_{2 n-1}\right), d\left(y_{2 n+1}, y_{2 n}\right), \frac{1}{2}\left(d\left(y_{2 n-1}, y_{2 n+1}\right)+d\left(y_{2 n}, y_{2 n}\right)\right)\right\}+\right. \\
& \left(1-a_{2}\right)\left\{\operatorname { m a x } \left(d^{2}\left(y_{2 n-1}, y_{2 n}\right), d\left(y_{2 n-1,} y_{2 n}\right) d\left(y_{2 n}, y_{2 n+1}\right), d\left(y_{2 n-1}, y_{2 n+1}\right) \cdot d\left(y_{2 n}, y_{2 n}\right),\right.\right. \\
& \left.\left.\left.\frac{d\left(y_{2 n}, y_{2 n-1}\right) d\left(y_{2 n}, y_{2 n}\right)}{2}, \frac{d\left(y_{2 n}, y_{2 n+1}\right) d\left(y_{2 n-1,} y_{2 n+1}\right)}{2}\right)\right\}^{1 / 2}\right] \\
& =\frac{a_{1}}{k^{4}}\left[a _ { 2 } \operatorname { m a x } \left\{d\left(y_{2 n-1}, y_{2 n}\right), d\left(y_{2 n+1}, y_{2 n}\right), \frac{1}{2}\left(d\left(y_{2 n-1}, y_{2 n+1}\right)\right\}+\right.\right. \\
& \left.\left(1-a_{2}\right)\left\{\max \left(d^{2}\left(y_{2 n-1}, y_{2 n}\right), d\left(y_{2 n-1,} y_{2 n}\right) d\left(y_{2 n+1}, y_{2 n}\right), \frac{d\left(y_{2 n}, y_{2 n+1}\right) d\left(y_{2 n-1,} y_{2 n+1}\right)}{2}\right)\right\}^{1 / 2}\right]
\end{aligned}
$$


$\leq \frac{a_{1}}{k^{4}}\left[a_{2} \max \left\{d\left(y_{2 n-1}, y_{2 n}\right), d\left(y_{2 n+1}, y_{2 n}\right), \frac{k}{2}\left(d\left(y_{2 n-1}, y_{2 n}\right)+d\left(y_{2 n}, y_{2 n+1}\right)\right\}+\right.\right.$

$\left.\left(1-a_{2}\right)\left\{\max \left(d^{2}\left(y_{2 n-1}, y_{2 n}\right), d\left(y_{2 n-1,} y_{2 n}\right) d\left(y_{2 n+1}, y_{2 n}\right), \frac{d\left(y_{2 n}, y_{2 n+1}\right) k\left(d\left(y_{2 n-1}, y_{2 n}\right)+\left(y_{2 n}, y_{2 n+1}\right)\right)}{2}\right)\right\}^{1 / 2}\right]$

$\left(y_{2 n}, y_{2 n+1}\right)>d\left(y_{2 n-1}, y_{2 n}\right)$, for some $n$ then from above inequality we have

$d\left(y_{2 n}, y_{2 n+1}\right)<\frac{a_{1}}{k^{4}}\left[a_{2} k d\left(y_{2 n}, y_{2 n+1}\right)+\left(1-a_{2}\right)\left\{k d^{2}\left(y_{2 n}, y_{2 n+1}\right)\right\}^{1 / 2}\right]$

$d\left(y_{2 n}, y_{2 n+1}\right)<\frac{a_{1}}{k^{4}}\left[a_{2} k d\left(y_{2 n}, y_{2 n+1}\right)+\left(1-a_{2}\right)\left\{k d\left(y_{2 n}, y_{2 n+1}\right)\right\}\right]$

$\left.=\frac{a_{1}}{k^{4}}\left[k d\left(a_{2}+\left(1-a_{2}\right)\right) d\left(y_{2 n}, y_{2 n+1}\right)\right\}\right]$

$=\frac{a_{1}}{k^{3}} d\left(\left(y_{2 n}, y_{2 n+1}\right)\right.$

$d\left(y_{2 n}, y_{2 n+1}\right)<\frac{a_{1}}{k^{3}} d\left(y_{2 n}, y_{2 n+1}\right)$

Which is a contradiction;

Hence $d\left(y_{2 n}, y_{2 n+1}\right) \leq d\left(y_{2 n-1}, y_{2 n}\right)$ for all $n \in N$ also by the above inequality we have

(2.2)d( $\left.y_{2 n}, y_{2 n+1}\right) \leq \frac{a_{1}}{k^{3}} d\left(y_{2 n-1}, y_{2 n}\right)$

$(2.3) d\left(y_{2 n-1}, y_{2 n}\right) \leq \frac{a_{1}}{k^{3}} d\left(y_{2 n-2}, y_{2 n-1}\right)$

From (2.2) and (2.3) we have

$d\left(y_{n}, y_{n-1}\right) \leq \lambda d\left(y_{n-1}, y_{n-2}\right)$

Where $\lambda=\frac{a_{1}}{k^{3}}<1$ and $\mathrm{n} \geq 2$, hence for all $\mathrm{n} \geq 2$ we obtain

$\mathrm{d}\left(\mathrm{y}_{\mathrm{n}}, \mathrm{y}_{\mathrm{n}-1}\right) \leq \ldots \ldots \ldots \ldots \ldots . \lambda^{\mathrm{m}-1} \mathrm{~d}\left(\mathrm{y}_{1}, \mathrm{y}_{0}\right) \quad \ldots \ldots \ldots$ (2.4), so for all $\mathrm{n}>\mathrm{m}$ we have

$\mathrm{d}(\mathrm{yn}, \mathrm{ym}) \leq \mathrm{kd}\left(\mathrm{y}_{\mathrm{m}}, \mathrm{y}_{\mathrm{m}+1}\right)+\mathrm{k}^{2} \mathrm{~d}\left(\mathrm{y}_{\mathrm{m}+1}, \mathrm{y}_{\mathrm{m}+2}\right)+\ldots \ldots \ldots \ldots \ldots+\mathrm{k}^{\mathrm{n}-\mathrm{m}-1} \mathrm{~d}\left(\mathrm{y}_{\mathrm{n}-1}, \mathrm{y}_{\mathrm{n}}\right)$.

from (2.4) we have 


$$
\begin{aligned}
\mathrm{d}\left(\mathrm{y}_{\left.\mathrm{n}, \mathrm{y}_{\mathrm{m}}\right)} \leq\right. & \leq\left(\mathrm{k} \lambda^{\mathrm{m}}, \mathrm{k}^{2} \lambda^{\mathrm{m}+1}+\ldots \ldots \ldots \ldots+\mathrm{k}^{\mathrm{n}-\mathrm{m}-1} \lambda^{\mathrm{n}-1}\right) \mathrm{d}\left(\mathrm{y}_{1}, \mathrm{y}_{0}\right) \\
& =\mathrm{k} \lambda^{\mathrm{m}}\left(1+\mathrm{k} \lambda+(\mathrm{k} \lambda)^{2}+\ldots \ldots \ldots\right) \mathrm{d}\left(\mathrm{y}_{1}, \mathrm{y}_{0}\right) \\
& \leq \frac{k \lambda^{m}}{1-k \lambda} d\left(y_{1}, y_{0}\right)
\end{aligned}
$$

On taking as $\mathrm{m}, \dot{\mathrm{n}} \rightarrow \infty$ we have $\mathrm{d}\left(\mathrm{y}_{\mathrm{n}}, \mathrm{y}_{\mathrm{m}}\right) \rightarrow 0$ as $\mathrm{k} \lambda<1$, therefore $\left\{\mathrm{y}_{\mathrm{n}}\right\}$ is a Cauchy sequence, since $\mathrm{X}$ is a complete $\mathrm{b}$-metric space, $\mathrm{B}$ some $\mathrm{y}$ in $\mathrm{X}$ such that .

$\lim \mathrm{n} \rightarrow \infty \mathrm{Fx}_{2 \mathrm{n}}=\lim \mathrm{n} \rightarrow \infty \mathrm{Tx}_{2 \mathrm{n}+1}=\lim \mathrm{n} \rightarrow \infty \mathrm{Gx}_{2 \mathrm{n}+1}=\lim \mathrm{n} \rightarrow \infty \mathrm{Sx}_{2 \mathrm{n}+2}=\mathrm{y}$

Since $S$ is continuous, therefore $\lim n \rightarrow \infty \mathrm{S}^{2} \mathrm{x}_{2 \mathrm{n}+2}=\operatorname{Sy}$ and $\lim \mathrm{n} \rightarrow \infty \mathrm{SFx}_{2 \mathrm{n}}=$ Sy and $\mathrm{STx}_{2 \mathrm{n}+1}=\mathrm{Sy}$

Since a pair $\{F, S\}$ is compatible, $\lim n \rightarrow \infty d\left(F^{2} x_{2 n}, S F x 2 n\right)=0$ so by lemma (1.5) we have

$\lim \mathrm{n} \rightarrow \infty \mathrm{FSx}_{2 \mathrm{n}}=\mathrm{Sy}$

Putting $\mathrm{x}=\mathrm{Sx}_{2 \mathrm{n}}$ and $\mathrm{y}=\mathrm{x}_{2 \mathrm{n}+1}$ in $(2.1)$

$(2.5) d\left(F S x_{2 n}, G x_{2 n+1}\right) \leq \frac{a_{1}}{k^{4}}\left[a_{2} \max \left\{d\left(S^{2} x_{2 n}, T x_{2 n+1}\right), d\left(F S x_{2 n}, S^{2} x_{2 n}\right), d\left(G x_{2 n+1}, T x_{2 n+1}\right), \frac{1}{2}\left(d\left(S^{2} x_{2 n}, G x_{2 n+1}\right)+\right.\right.\right.$ $\left.\left.d\left(F S x_{2 n}, T x_{2 n+1}\right)\right)\right\}+\left(1-a_{2}\right)\left\{\max \left(d^{2}\left(S^{2} x_{2 n}, T x_{2 n+1}\right), d\left(S^{2} x_{2 n}, F S x_{2 n}\right) d G x_{2 n+1}, T x_{2 n+1}\right)\right.$,

$\left.\left.\left.d\left(S^{2} x_{2 n}, G x_{2 n+1}\right) \cdot d\left(F S x_{2 n}, T x_{2 n+1}\right), \frac{d\left(F S x_{2 n}, S^{2} x_{2 n}\right) d\left(F S x_{2 n}, S x_{2 n+1}\right)}{2}, \frac{d\left(S x_{2 n+1}, G x_{2 n+1}\right) d\left(S^{2} x_{2 n}, G x_{2 n+1}\right)}{2}\right)\right\}^{1 / 2}\right]$

Taking upper limit as $\mathrm{n} \rightarrow \infty$ in above and using lemma (1.4)

$$
\begin{aligned}
& \frac{d(S y, y)}{k^{2} \leq} \lim n \rightarrow \infty \sup d\left(F S x_{2 n}, G x_{2 n+1}\right) \leq \frac{a_{1}}{k^{4}}\left[a_{2} \max \left\{k^{2} d(S y, y), \frac{k^{2}}{2}(d(S y, y)+d(S y, y))\right\}+\right. \\
& \left.\left(1-a_{2}\right)\left\{\max \left(d^{2}(S y, y), d(S y, S y) d(y, y), d(S y, y) \cdot d(S y, y), \frac{d(S y, S y) d(S y, y)}{2}, \frac{d(y, y) d(S y, y)}{2}\right)\right\}^{1 / 2}\right] \\
\leq & \frac{a_{1}}{k^{4}}\left[a_{2} k^{2} d(S y, y)+\left(1-a_{2}\right)\left\{\max \left(d^{2}(S y, y), d^{2}(S y, y)\right)\right\}^{1 / 2}\right] \\
\leq & \frac{a_{1}}{k^{4}}\left[a_{2} k^{2} d(S y, y)+\left(1-a_{2}\right)\left\{k^{4}\left(d^{2}(S y, y)\right\}^{1 / 2}\right]\right. \\
\leq & \left.\frac{a_{1}}{k^{4}}\left[a_{2} k^{2} d(S y, y)+\left(1-a_{2}\right) k^{2} d(S y, y)\right\}\right] \\
\leq & \frac{a_{1}}{k^{4}}\left[k^{2} d(S y, y)\left(a_{2}+1-a_{2}\right)\right] \\
\leq & \frac{a_{1}}{k^{4}}\left[k^{2} d(S y, y)\left(a_{2}+1-a_{2}\right)\right] \\
\leq & \frac{a_{1}}{k^{2}} d(S y, y)
\end{aligned}
$$


$\frac{d(S y, y)}{k^{2}} \leq \frac{a_{1}}{k^{2}} d(S y, y)$

$d($ Sy,y $) \leq \mathrm{a}_{1} \mathrm{~d}(\mathrm{Sy}, \mathrm{y})$ and $\mathrm{a}_{1} \in(0,1)$

$\Rightarrow S y=y$

Put $\mathrm{x}=\mathrm{x}_{2 \mathrm{n}}, \mathrm{y}=\mathrm{Tx}_{2 \mathrm{n}+1}$ in (2.1) and using continuity of $\mathrm{T}$

$\lim n \rightarrow \infty T^{2} x_{2 n+1}=T y$ and $T G x_{2 n+1}=T y$ and Since a pair $\{G, T\}$ is compatible then

$\lim n \rightarrow \infty d\left(\mathrm{GTx}_{\mathrm{n}}, \mathrm{TGx}_{\mathrm{n}}\right)=0$

So by lemma (1.5) we have $\lim n \rightarrow \infty \mathrm{GTx}_{2 \mathrm{n}}=\mathrm{Ty}$

$(2.6) d\left(F x_{2 n}, G T x_{2 n+1}\right) \leq \frac{a_{1}}{k^{4}}\left[a_{2} \max \left\{d\left(S x_{2 n}, T^{2} x_{2 n+1}\right), d\left(F x_{2 n}, S x_{2 n}\right), d\left(G T x_{2 n+1}, T^{2} x_{2 n+1}\right), \frac{1}{2}\left(d\left(S x_{2 n}, G T x_{2 n+1}\right)+\right.\right.\right.$ $\left.\left.d\left(F x_{2 n}, T^{2} x_{2 n+1}\right)\right)\right\}+\left(1-a_{2}\right)\left\{\max \left(d^{2}\left(S x_{2 n}, T^{2} x_{2 n+1}\right), d\left(S x_{2 n}, F x_{2 n}\right) d G T x_{2 n+1}, T^{2} x_{2 n+1}\right)\right.$

$\left.\left.\left.d\left(S x_{2 n}, G T x_{2 n+1}\right) \cdot d\left(F x_{2 n}, T^{2} x_{2 n+1}\right), \frac{d\left(F x_{2 n}, S x_{2 n}\right) d\left(F x_{2 n}, S T x_{2 n+1}\right)}{2}, \frac{d\left(S T x_{2 n+1}, G T x_{2 n+1}\right) d\left(S x_{2 n}, G T x_{2 n+1}\right)}{2}\right)\right\}^{1 / 2}\right]$

Taking upper limit as $n \rightarrow \infty$ in above and using lemma (1.4) we obtain

$$
\begin{aligned}
& \frac{d(y, T y)}{k^{2}} \leq \lim n \rightarrow \infty \sup d\left(F x_{2 n}, G T x_{2 n+1}\right) \leq \frac{a_{1}}{k^{4}}\left[a_{2} \max \left\{k^{2} d(y, T y), \frac{k^{2}}{2}(d(y, T y)+d(y, T y))\right\}+\right. \\
& \left.\left(1-a_{2}\right)\left\{\max \left(d^{2}(y, T y), d(y, y) d(T y, T y), d(y, T y) \cdot d(y, T y), \frac{d(y, y) d(y, S y)}{2}, \frac{d(S y, T y) d(y, T y)}{2}\right)\right\}^{1 / 2}\right] \\
& \leq \frac{a_{1}}{k^{4}}\left[a_{2} k^{2} d(y, T y)+\left(1-a_{2}\right)\left\{\max \left(k^{4} d^{2}(y, T y), d^{2}(y, T y), \frac{k^{4} d^{2} d(y, T y)}{2}\right)\right\}^{1 / 2}\right] \\
& \leq \frac{a_{1}}{k^{4}}\left[a_{2} k^{2} d(y, T y)+\left(1-a_{2}\right) k^{2} d(y, T y)\right] \\
& \leq \frac{a_{1}}{k^{4}}\left[\left(a_{2}+1-a_{2}\right) k^{2} d(y, T y)\right] \\
& \left.\leq \frac{a_{1}}{k^{2}} d(y, T y)\right] \\
& \frac{d(y, T y)}{k^{2}} \leq \frac{a_{1}}{k^{2}} d(y, T y)
\end{aligned}
$$$$
d(\mathrm{y}, \mathrm{Ty}) \leq \mathrm{a}_{1} \mathrm{~d}(\mathrm{y}, \mathrm{Ty}) \text { and } \mathrm{a}_{1} \in(0,1)
$$$$
\Rightarrow y=T y
$$ 
Put $\mathrm{x}=\mathrm{y}$ and $\mathrm{y}=\mathrm{x}_{2 \mathrm{n}+1}$ in $(2.1)$ then

$(2.7) d\left(F y, G x_{2 n+1}\right) \leq \frac{a_{1}}{k^{4}}\left[a_{2} \max \left\{d\left(S y, T x_{2 n+1}\right), d(F y, S y), d\left(G x_{2 n+1}, T x_{2 n+1}\right), \frac{1}{2}\left(d\left(S y, G x_{2 n+1}\right)+\right.\right.\right.$ $\left.\left.d\left(F y, T x_{2 n+1}\right)\right)\right\}+\left(1-a_{2}\right)\left\{\max \left(d^{2}\left(S y, T x_{2 n+1}\right), d(S y, F y) d\left(G x_{2 n+1}, T x_{2 n+1}\right)\right.\right.$, $\left.\left.\left.d\left(S y, G x_{2 n+1}\right) \cdot d\left(T x_{2 n+1}, F y\right), \frac{d(F y, S y) d\left(F y, S x_{2 n+1}\right)}{2}, \frac{d\left(S x_{2 n+1}, G x_{2 n+1}\right) d\left(S y, G x_{2 n+1}\right)}{2}\right)\right\}^{1 / 2}\right]$

Taking upper limit as $\mathrm{n} \rightarrow \infty$ in above and using lemma (1.9) and using $\mathrm{Sy}=\mathrm{Ty}=\mathrm{y}$ we have

$\frac{d(F y, y)}{k^{2}} \leq \frac{a_{1}}{k^{4}}\left[a_{2} \max \left\{k^{2} d(S y, y), k^{2} d(F y, S y), k^{2} d(y, y), \frac{k^{2}}{2}(d(S y, y)+d(F y, y))\right\}+\right.$ $\left.\left(1-a_{2}\right)\left\{\max \left(d^{2}(S y, y), d(S y, F y) d(y, y), d(S y, y) \cdot d(F y, y), \frac{d(F y, S y) d(F y, y)}{2}, \frac{d(y, y) d(S y, y)}{2}\right)\right\}^{1 / 2}\right]$

$\frac{d(F y, y)}{k^{2}} \leq \frac{a_{1}}{k^{4}}\left[\left\{a_{2} k^{2} d(F y, y)\right\}+\left(1-a_{2}\right)\left\{\max \left(k^{2} \cdot d(F y, y), \frac{k^{4} d^{2}(F y, y)}{2}\right)\right\}^{1 / 2}\right]$

$\frac{d(F y, y)}{k^{2}} \leq \frac{a_{1}}{k^{4}}\left[\left\{a_{2} k^{2} d(F y, y)\right\}+\left(1-a_{2}\right) k^{2} d(F y, y)\right]$

$\frac{d(F y, y)}{k^{2}} \leq \frac{a_{1}}{k^{4}}\left[d(F y, y) k^{2}\left(a_{2}+1-a_{2}\right)\right]$

$\left.\frac{d(F y, y)}{k^{2}} \leq \frac{a_{1}}{k^{2}} d(F y, y)\right]$ And $\mathrm{a}_{1} \in(0,1)$

$d(F y, y) \leq a_{1} d(F y, y)$

Which is a contradiction

$d(F y, y)=0$

$\Rightarrow y=F y \quad$ As $_{1} \in(0,1)$

So $S Y=T y=F y=Y$ we have

$(2.8) d(y, G y)=d(F y, G y) \leq \frac{a_{1}}{k^{4}}\left[a_{2} \max \left\{d(S y, T y), d(F y, S y), d(G y, T y), \frac{1}{2}(d(S y, G y)+\right.\right.$

$d(F y, T y))\}+\left(1-a_{2}\right)\left\{\max \left(d^{2}(S y, T y), d(S y, F y) d(G y, T y)\right.\right.$,

$\left.\left.\left.d(S y, G y) \cdot d(F y, T y), \frac{d(F y, S y) d(F y, S y)}{2}, \frac{d(S y, G y) d(S y, G y)}{2}\right)\right\}^{1 / 2}\right]$

$\leq \frac{a_{1}}{k^{4}}\left[a_{2} d(y, G y)+\left(1-a_{2}\right)\left\{\max \left(d^{2}(y, y), d(y, y) d(G y, y), d(y, G y) \cdot d(y, y)\right.\right.\right.$,

$\left.\left.\left.\frac{d(y, y) d(y, y)}{2}, \frac{d(y, G y) d(y, G y)}{2}\right)\right\}^{1 / 2}\right]$ 
$\leq \frac{a_{1}}{k^{4}}\left[a_{2} d(y, G y)+\left(1-a_{2}\right) d(y, G y)\right]$

$\leq \frac{a_{1}}{k^{4}}\left[d(y, G y)\left(a_{2}+1-a_{2}\right]\right.$

$$
d(y, G y) \leq a_{1} d(y, G y)
$$

Which is a contradiction as $\mathrm{a}_{1} \epsilon(0,1)$

$\Rightarrow d(y, G y)=0$

$\Rightarrow y=G y$

Uniqueness of $\mathbf{y}$ : if there exit another common fixed point $\mathrm{x}$ in $\mathrm{X}$ for $\mathrm{F}, \mathrm{G}, \mathrm{S}, \mathrm{T}$

$$
\begin{aligned}
& d(x, y)=d(F x, G y) \leq \frac{a_{1}}{k^{4}}\left[a _ { 2 } \operatorname { m a x } \left\{d(S x, T y), d(F x, S x), d(G y, T y), \frac{1}{2}(d(S x, G y)+\right.\right. \\
& \left.d(F x, T y))\}+\left(1-a_{2}\right)\left\{\max \left(d^{2}(x, y), d(x, x) d(y, y), d(x, y) \cdot d(x, y), \frac{d(x, x) d(x, y)}{2}, \frac{d(y, y) d(x, y)}{2}\right)\right\}^{1 / 2}\right] \\
& \leq \frac{a_{1}}{k^{4}}\left[a_{2} d(x, y)+\left(1-a_{2}\right)\left\{\max \left(d^{2}(x, y), d^{2}(x, y)\right\}^{1 / 2}\right]\right. \\
& \qquad \leq \frac{a_{1}}{k^{4}}\left[a_{2} d(x, y)+\left(1-a_{2}\right) d(x, y)\right] \\
& \leq \frac{a_{1}}{k^{4}} d(x, y)<a_{1} d(x, y) \\
& \Rightarrow d(x, y)<a_{1} d(x, y) \\
& \Rightarrow d(x, y)=0 \\
& \Rightarrow x=y
\end{aligned}
$$

Thus $y$ is a unique common fixed point of $F, G, S, T$.

\section{Example:}

Let $\mathrm{X}=[0, \infty)$ be endowed with b metric space $(\mathrm{X}, \mathrm{d})$ with metric $d(x, y)=|x-y|^{2}=(x-y)^{2}$, define $\mathrm{F}, \mathrm{G}, \mathrm{S}, \mathrm{T}$ on $\mathrm{X}$ by $F(x)=\log \left(1+\frac{x}{4}\right), G(x)=\log \left(1+\frac{x}{6}\right)$

$S(x)=e^{3 x}-1, T(x)=e^{2 x}-1$.

Obviously $F(x)=G(x)=T(X)=S(X)=[0, \infty)$.

We show that the pair $(F, S)$ is compatible: let $\left\{x_{n}\right\}$ be a sequence in $X$ such that for some $t \in X$ 
$\lim n \rightarrow \infty d\left(F_{n}, t\right)=0$ and $t \in X \lim n \rightarrow \infty d\left(\mathrm{Sx}_{n}, t\right)=0$

i.e $\lim \mathrm{n} \rightarrow \infty\left|F x_{n}-t\right|=0, \lim \mathrm{n} \rightarrow \infty\left|S x_{n}-t\right|=0$.since $\mathrm{F}$ and $\mathrm{S}$ are continuous , we have

$\lim n \rightarrow \infty \mathrm{d}\left(\mathrm{FSx}_{\mathrm{n}}, \mathrm{SFx}_{\mathrm{n}}\right)=\lim \mathrm{n} \rightarrow \infty\left|F S x_{n}-S F x_{n}\right|^{2}=|F t-S t|^{2}=\left|\log \left(1+\frac{t}{4}\right)-\left(e^{3 t}-1\right)\right|^{2}=0 \Leftrightarrow t=0$

Then $(\mathrm{F}, \mathrm{S})$ are compatible.

Similarly $\{\mathrm{G}, \mathrm{T}\}$ are compatible.

For each $\mathrm{x}, \mathrm{y} \in \mathrm{X}$

$$
\begin{gathered}
\mathrm{d}(\mathrm{Fx}, \mathrm{Gy})=(\mathrm{Fx}-\mathrm{Gy})^{2}=\left[\log \left(1+\frac{x}{4}\right)-\log \left(1+\frac{y}{6}\right)\right]^{2} \leq\left(\frac{x}{4}-\frac{y}{6}\right)^{2} \leq \frac{1}{(12)^{2}}\left[(3 x-2 y)^{2}\right] \\
\left.\leq \frac{1}{(12)^{2}}\left[e^{3 x}-e^{2 y}\right)\right]^{2} \\
=\frac{1}{144}[d(S x, T y)]
\end{gathered}
$$

$$
\begin{aligned}
& \leq \frac{1}{2^{4}}\left[a_{2} \max \left\{d(S x, T y), d(S x, S x), d(T y, T y), \frac{1}{2}(d(S x, T y)+d(S x, T y))\right\}+\left(1-a_{2}\right)\left\{\operatorname { m a x } \left(d^{2}(S x, T y),\right.\right.\right. \\
& \left.\left.\left.d(S x, S x) d(T y, T y), d(S x, T y) \cdot d(S x, T y), \frac{d(S x, S x) d(S x, S y)}{2}, \frac{d(S y, T y) d(S x, T y)}{2}\right)\right\}^{1 / 2}\right]
\end{aligned}
$$

Where $a_{1}=\frac{1}{9} \in(0,1)$ and $\mathrm{k}=2$

Thus F,G,S,T satisfy all condition of theorem (2.1) .moreover 0 is the unique common fixed point of F,G,S,T.

\section{References}

[1]. Akkouchi M., Common fixed point theorems for two self-mapping of a b-metric space under an implicit relation, Hacettepe Journal of mathematics and statistics , 40 (6),805- 810 ,2011

[2]. Aydi ,H.Bota ,M.Karapinar,E.and Moradi,S.A ,fixed point for weak $\Phi$-contractions on b- metric spaces, Fixed point theory, 13 (2) , $337-346$, 2012.

[3] . Bakhtin, I.A. , Contraction mapping principle in almost metric spaces ,Funct. Anal.30, 26-37,1989.

[4] Boriceanu, M.,Fixed point theory for multivalued generalized contraction on a set with two b-metric, Studia, Univ Babes, Bolya: Math, Liv(3) ,1-14,2009.

[5].Bota, M., Molnar, A. and Varga, C.S , On ekeland's variational principle in b-metric spaces, Fixed Point Theory 12(2), 21-28,2011.

[6]. Bourbaki, N., Topologie Generale; Herman: Paris, France, 1974.

[7]. Ciric , L.B.,A generalization of Banach's contraction principle,proceedings of the American Mathematical Society, 45 , $267-273$, 1974.

[8]. Czerwik S, Contraction mappings in b-metric spaces, Acta Math. Inform. Univ. Ostra., 1, 5-11, 1993.

[9].Czerwik, S., Non-linear set valued contraction mappings in b-metric spaces, Atti sem math fiq Univ. Modena, 46 (2 ),263-276,1998.

[10]. J R. Roshan , N.shobkolaei, S.sedghiand M.abbas, Common fixed point of four maps in b- Metric spaces , Hacettepe Journal of Mathematics and Statistics , 4(4),613-624,2014.

[11] .Kir, Mehmet, Kiziltune, Hukmi, On some well-known fixed point theorems in b-metric space, Turkish journal of analysis and number theory, 1( 1) , 13-16, 2013.

[12]. Pacurar, M.Sequences of almost contractions and fixed points in b-metric spaces, Analele Universităţ de Vest, Timisoara, Seria Matematică Informatică, $3,125-137,2010$.

[13]. Shah, M.H.and Hussain , N. Nonlinear contractions in partially ordered quasi b-metric spaces, Commun. Korean Math. Soc. 27(1), 117-128 , 2012. 